\title{
Visual Outcomes with Contact Lenses Previous Keratoplasty
}

\section{Gonzalo Carracedo}

\begin{abstract}
We describe a case of a 22-year-old patient, with severe keratoconus in both eyes. Penetrating keratoplasty in left eye was performed in August 2011. The patient was awaiting postoperative stabilization of this eye prior to surgery on the right eye. Therefore, a Clearkone ${ }^{\circledR}$ hybrid contact lens was fitted on the right eye to maintain corneal epithelial integrity, improve quality of vision and therefore qualify of life during this interim period. Visual acuity with the contact lens was +0.1 logMAR. Comfort was excellent and the patient was able to wear the Clearkone ${ }^{\circledR}$ lens up to 12 hours per day. Similar outcomes regarding vision and comfort were obtained at follow-up visits through 9 months. In conclusion, new hybrid contact lens designs for keratoconus are an excellent option for vision rehabilitation in severe cases of keratoconus which would otherwise be left with low vision for several months while waiting for keratoplasty.
\end{abstract}

Keywords: Contact lenses, Hybrid contact lenses, Keratoplast.

How to cite this article: Carracedo G. Visual Outcomes with Contact Lenses Previous Keratoplasty. Int J Kerat Ect Cor Dis 2012;1(3):196-200.

\section{Source of support $\mathrm{Nil}$}

Conflict of interest: None declared

\section{INTRODUCTION}

Keratoconus is a progressive, asymmetric dystrophy of the cornea characterized by steepening and distortion of the cornea, apical thinning and central scarring. It is generally bilateral and progresses asymmetrically in both eyes of the individual. It is usually treated with contact lenses before other surgical procedures, such as penetrating keratoplasty (PKP), which is normally reserved for cases of contact lens intolerance or poor contact lens vision. ${ }^{1}$ The most frequently used contact lenses to compensate irregular astigmatism that is a hallmark of keratoconus are rigid gas permeable (RGP) lenses with conventional spherical, aspheric optics as well as scleral lenses or hybrid lenses with an RGP optic and soft skirt. ${ }^{2-5}$

Contact lens intolerance is one of the main reasons for recommending surgery in keratoconus patients. W hile they are often the lens of choice, RGP contact lenses can often cause significant discomfort leading to limited wear time. Furthermore, adaptation with them is complicated as the severity of keratoconus progresses. ${ }^{1}$ W hen contact lenses cannot be successfully fitted, most keratoconus patients seek PK P surgery or other procedures, such as corneal ring surgery or corneal-collagen crosslinking to stabilize the keratoconus and maintain vision. ${ }^{6,7} \mathrm{~N}$ ew R GP scleral contact lens designs can improve patient tolerance but they are still associated with problems common to scleral lenses, such as warping and seal-off, leading to discomfort and corneal dystrophy. We present data on an alternative to traditional R GP contact lenses or scleral lenses; namely a hybrid contact lens with a RGP optic that optimized visual outcomes which is combined with a soft (HEM A) skirt that improves comfort and wearability for the patient being a good opportunity for patients with advanced keratoconus. ${ }^{5,8}$

\section{CASE REPORT}

A 22-year-old patient was referred to the 0 ptometric Clinic in the U niversity of Complutense (M adrid, Spain) for contact lens fitting in the right eye in October 2011. The patient had previously undergone PK P in the left eye (A ugust 2011, approximately 3 months earlier) and had been waiting for full healing of this surgery before proceeding with fitting of a contact lens in the right eye. Contact lens fitting in the left eye was not considered at this time until removal of sutures which was not yet scheduled.

The patient wore R GP contact lenses 5 years prior but discontinued wearing them because of discomfort and difficulty in maintaining the lenses (patient lost several lenses over time). The patient reported no need to rub eyes as is frequently indicated by other patients with keratoconus. ${ }^{1}$ A lso, he reported no presence of any history of atopic and connective disease but he has a family history of keratoconus.

The patient had an uncorrected visual acuity (UCVA) of $+1.3 \log$ M A R ( 0.05 Snellen) visual acuity measured at 3 meters for right eye and $+1.0 \log M A R$ ( 0.10 Snellen) visual acuity at 6 meters in the left eye. $V$ isual acuity with pinhole test improved to $0.2 \log M A R(0,63)$ in both eyes. The manifest refraction was -7.00 sphere, -14.00 cylinder at an axis of $160^{\circ}$ and +4.00 sphere, -6.00 cylinder at an axis of 1450 for right eye and left eyes respectively. Best spectacle corrected visual acuity (BSCVA) was +0.8 $\log M A R(0.16)$ visual acuity for the right eye and +0.5 logM AR (0.32 Snellen) visual acuity for the left eye.

Corneal topography in right eye showed a severe keratoconus, with a steep keratometry radius of 76.19 Diopter and with a large extension. The corneal astigmatism is similar to the one we obtained in the manifest refraction 
(Fig. 1). In addition, the high order aberration RM S is quite high, over $6 \mu \mathrm{m}$. Slit-lamp examination showed severe corneal thinning (Fig. 2), central leucoma (Fig. 3) and Vogt striae (Fig. 4). B ased on the A msler-K rumeich scale this patient presents with keratoconus grade IV. On the other hand, and according to KSS scale, ${ }^{9}$ the keratoconus is grade $V$. In any case, both values represent the most severe grade of keratoconus in each scale.

In order to improve patient vision, we chose a contact lens that could be fit without bearing on the cone apex, in order to preserve the corneal integrity. The chosen contact lens was a hybrid contact lens design, specific for keratoconus with an RGP center (paflufocon D) and a soft HEMA skirt (hemiberfilcon A; Clearkone ${ }^{\circledR}$, SynergEyes Inc., Carlsbad, CA, USA).

The fitting of the Clearkone ${ }^{\circledR}$ is based on the concept of sagittal depth setting in relation to the irregular cornea. The fit of the Clearkone ${ }^{\circledR}$ depends upon the depth of the lens clearing the elevation of the cone, first determining the minimal vault needed to clear the cone and then, determining the skirt curvature that fits onto the sclera. The aim is to determine the appropriate vault that provides complete apical clearance to clear the cone. The fit of the vault is independent of the fit of the skirt curvature, and in this sense, each should be fitted separately.

The first lens fitted was vault 550, Skirt Medium and power $-11.00 \mathrm{D}$. W ith this lens, the patient achieved a visual acuity of $+0.1 \log$ M A R (0.8 Snellen). This lens had a touch in the mid-peripheral cornea, at the junction of the optic and skirt materials which the manufacturer calls the inner landing zone (ILZ; Fig. 5). The patient reported discomfort with this lens so we changed to a steeper skirt parameter while maintaining the fitted vault at $550 \mu$ and the power at $-11.00 \mathrm{D}$. The visual acuity remained the same as with the first lens but since the steeper skirt allowed the lens to avoid the ILZ bearing, comfort was increased. In Figure 6 we can see the sufficient apical clearance to prevent the bearing in the apex. A fter 9 months, the patient was able to wear the lens for 12 hours per day with no report of any discomfort. No evidence of erosion either at the apex or in the corneal periphery was present and there are no signs of dystrophy which might lead to neovascularization. Prior to discharge, we suggested the patient use of multipurpose solution with hyaluronate (i.e. BioT rue, B\&L, R ochester, USA) to reinforce comfort. Also, for cleaning purposes we indicated an isopropyl alcohol cleaner solution (i.e. Duolens, Lenticon, Spain).

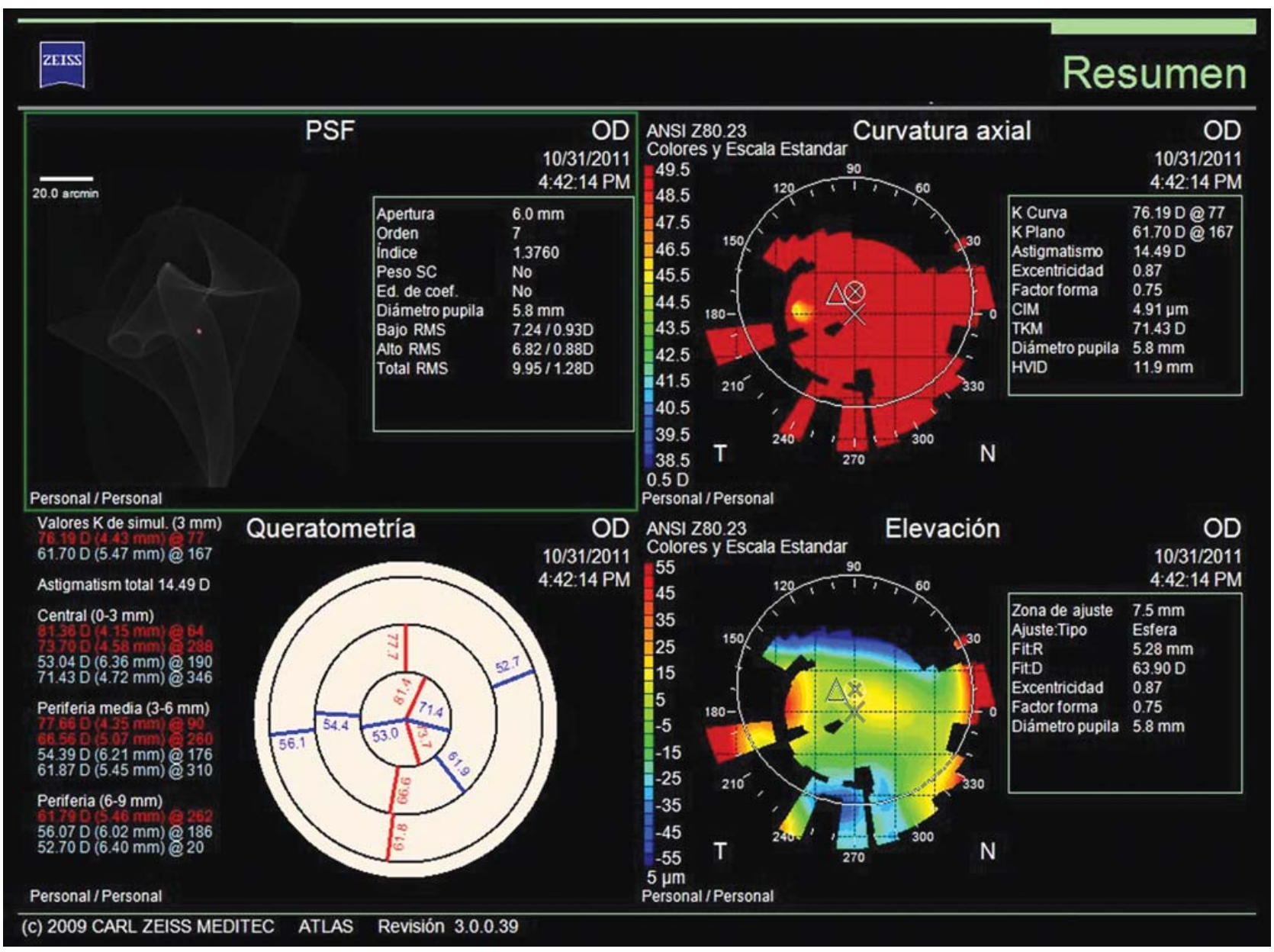

Fig. 1: Corneal topography of OD 


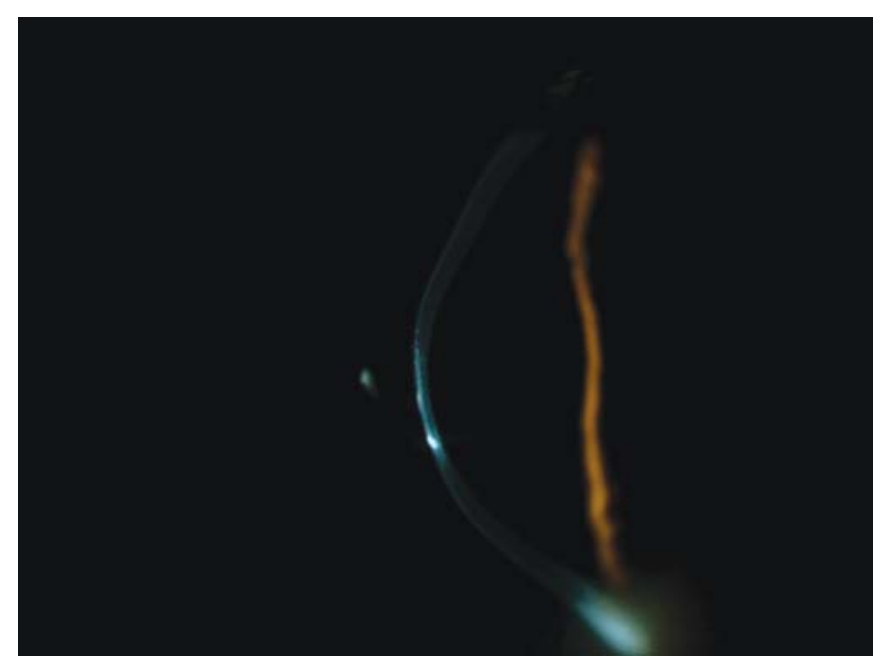

Fig. 2: Slit-lamp photography showing corneal thinning in OD. The pachymetry was of $264 \mu \mathrm{m}$

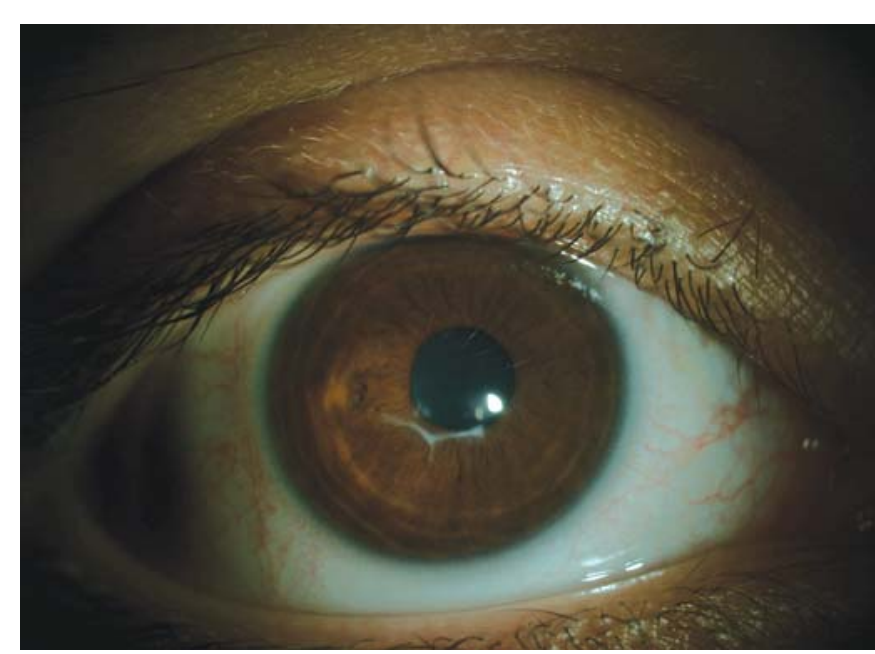

Fig. 3: Slit-lamp photography showing central leucoma

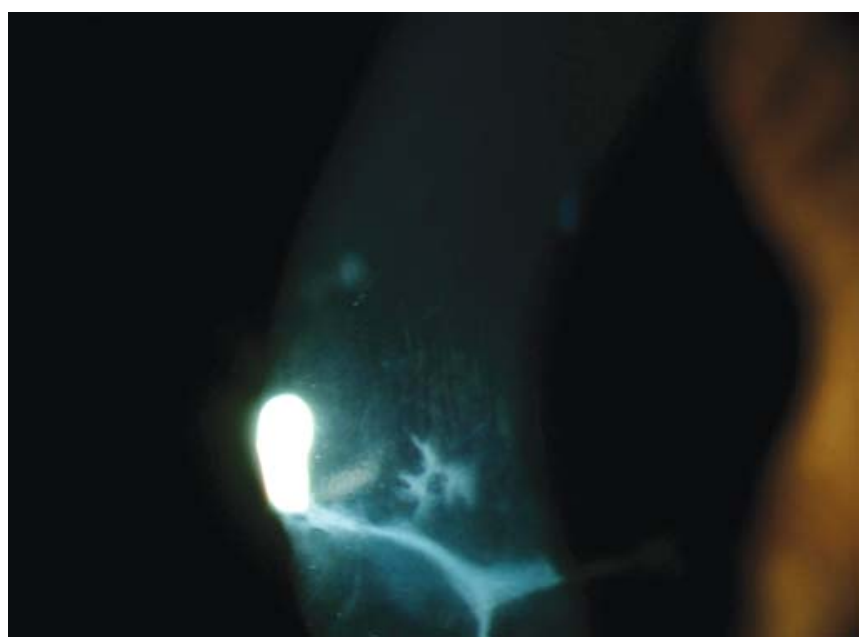

Fig. 4: Slit-lamp photography showing leucoma and Vogt's striae

The patient was examined in the hospital at 3, 6 and 9 months after fitting to assess the progression of the keratoconus, and in the optometry clinic, to evaluate vision and contact lens fitting. We found no significant changes in the right eye.

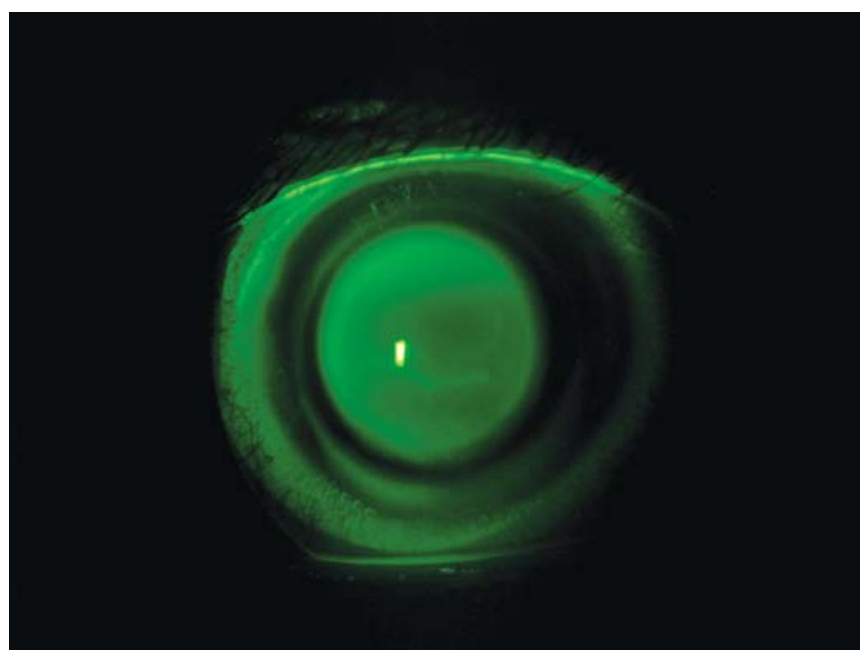

Fig. 5: Slit-lamp photograph showing fluorogram pattern of ClearKone ${ }^{\circledR}$ lens with bearing in ILZ

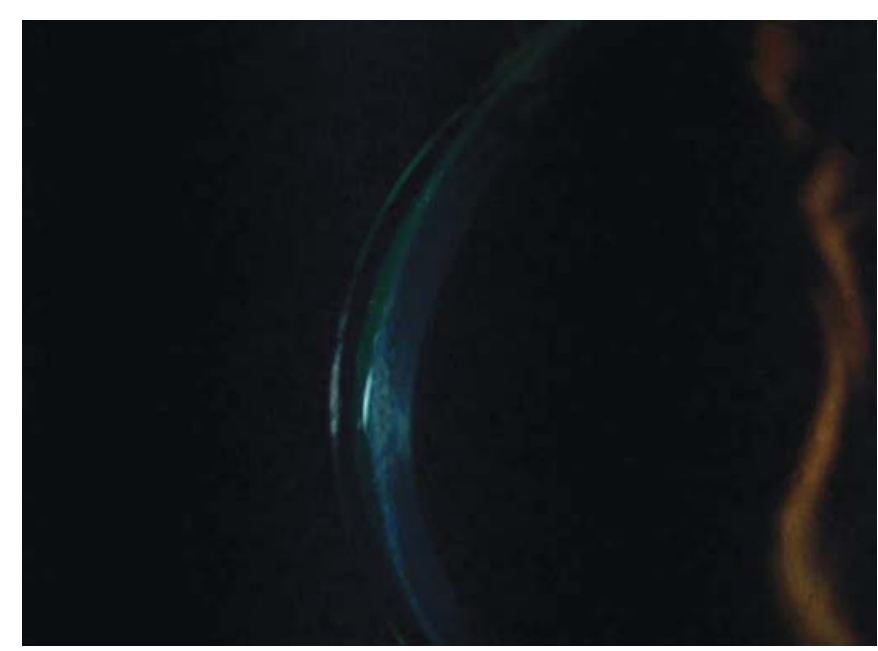

Fig. 6: Slit-lamp photography showing a clearance between the cornea and lens to avoid any touch in the cornea

\section{DISCUSSION}

It has been estimated that around $12 \%$ of keratoconus patients eventually require PKP. ${ }^{10}$ Postoperative visual rehabilitation after keratoplasty usually results in wearing glasses for most patients, but to obtain the optimal vision, they need to wear contact lenses. ${ }^{11}$ A fter selective suture removal following keratoplasty, contact lens fitting should be considered. Some studies suggest that the appropriate time between keratoplasty and initial contact lens fitting for these should be between 8 and 18 months. ${ }^{12,13}$ During this time, the patients probably have limited vision with the treated eye and therefore they need the excellent vision in the other eye. In such cases, fitting the nonoperated eye with a contact lens is the best option.

RGP lenses are the most commonly prescribed contact lenses by optometrists for eyes with keratoconus. $\mathrm{N}$ evertheless, this is not the only possibility. There are other possible designs, such as thicker contact lenses, scleral 
lenses and hybrid lenses. There are specialized soft contact lens designs for keratoconus with greater central thickness, being the aim to compensate higher order aberrations than standard soft contact lenses. The main benefit of these is the excellent comfort compared with RGP Ienses. ${ }^{16}$ In this case, we decided not to use this design because of the patient's high RMS, which would not be compensate for with thicker soft contact lenses, and therefore would not result in good visual acuity.

There are three general viewpoints when fitting gas permeable contact lenses in eyes with keratoconus: (i) Fitting with three-point touch, (ii) fitting with apical bearing but with minimal peripheral stabilization and (iii) Fitting with full apical clearance. M ost of optometrists favor the first technique. The goal in three-point touch is to achieve a mild apical touch and then mid-peripheral touch on the slopes of the cone to distribute lens bearing/pressure across a relatively large area so as to reduce excessive pressure in the apex. There are many studies about the efficacy and effectiveness of these types of lenses, providing a good vision but a poor comfort.1,14

We decided against fitting gas permeable lenses in this patient for various reasons. For our patient described here, it was critical to improve the visual acuity of the nonsurgical eye because the best visual acuity with spectacles with in the surgical eye (left eye) was very poor. The challenge in this case was to fit a contact lens in an eye with a severe keratoconus despite the poor corneal integrity. Some studies describe an increase in inflammatory molecules in gas permeable lens wearers with keratoconus, and these increased levels are higher in cases with severe keratoconus. ${ }^{15}$ With that in mind, our primary goal was to avoid apical bearing, which could have provoked complications, such as epithelial integrity. Secondly, but also importantly, the patient previously wore and rejected RGP lenses due to discomfort so a new lens type was needed.

Concerning soft contact lenses for keratoconus, there is a special design with more central thickness, being the aim to compensate higher order aberrations than standard soft contact lenses. The main feature is the excellent comfort compared with gas permeable lenses. ${ }^{16}$ In our case, we decided not to use this design because the patient had a high RMS, which would not compensate for thicker soft contact lenses, and therefore he would not achieve good visual acuity.

Scleral contact lenses are large-diameter gas permeable lenses with diameters varying from $13 \mathrm{~mm}$ to more than $20 \mathrm{~mm}$. Unlike standard RG P lenses, scleral contact lenses completely cover the cornea and extend onto the sclera and therefore can provide better initial comfort, centration and stability compared to a corneal RGP lens and better vision than would be achieved with thicker soft contact lenses. ${ }^{17}$ So, for this patient, a scleral lens with a diameter of 16.50 to $18.00 \mathrm{~mm}$ to optimize the fit would usually be the lens design of choice fit. N evertheless, we decided to fit a hybrid lens because of its smaller less diameter than the scleral lenses. This facilitated easier insertion and removal of the lens while providing vision and comfort similar to a scleral lens.

Hybrid contact lens provides excellent visual acuity improvement in patients with moderate-to-severe corneal ectasia, but despite the good clinical performance in terms of visual rehabilitation, clinicians should be aware of the potential complications with this fitting approach. These include corneal indentation in the transition zone between the rigid and the soft material, typically associated with a loss of vaulting over time. Although the cause-effect relationship has not been established, this could be partially responsible for severe complications, such as severe keratitis or edema. ${ }^{8,18}$ Clinicians must get specific training to fit the lens and to be aware of some potential complications. In this case, we were able to fit this patient with a new type of lens with excellent results.

\section{CONCLUSION}

The new hybrid contact lens designs that combine a rigid RGP center with a soft skirt are an excellent option for vision rehabilitation in patients with severe keratoconus, which would otherwise be forced to endure low vision for several months prior to keratoplasty. In most of cases, with these contact lenses, we can delay or perhaps prevent corneal transplants in keratoconic eyes with contact lens intolerance. In our case, we have succeeded to improve the quality of vision until the keratoplasty and therefore, the quality of life of the patient.

\section{REFERENCES}

1. Romero-J imenez M, Santodomingo-Rubido J, Wolffsohn J S. K eratoconus: A review. Cont Lens Anterior Eye 2012 A ug;33(4):157-166.

2. $Y$ anai $R$, U eda $K, N$ ishida $T$. R etrospective analysis of vision correction and lens tolerance in keratoconus patients prescribed a contact lens with dual aspherical curves. Eye Contact Lens $2010 \mathrm{M}$ ar;36(2):86-89.

3. Jinabhai A, N eil Charman W, O' Donnell C, Radhakrishnan H. O ptical quality for keratoconic eyes with conventional RGP lens and simulated, customised contact lens corrections: A comparison. Ophthalmic Physiol Opt 2011 M ay;32(3):200-12.

4. A bdalla Y F, Elsahn A F, Hammersmith K M , Cohen EJ. Synerg eyes lenses for keratoconus. Cornea 2010 Jan;29(1):5-8.

5. Barnett $M, M$ annis $M J$. Contact lenses in the management of keratoconus. Cornea $2011 \mathrm{Dec} ; 30(12): 1510-16$.

6. Kymionis GD, Siganos CS, Tsiklis NS, et al. Long-term followup of Intacs in keratoconus. Am J Ophthalmol $2007 \mathrm{Feb}$; $143(2): 236-44$. 
7. Wollensak G, Spoerl E, Seiler T. Riboflavin/ultraviolet-ainduced collagen crosslinking for the treatment of keratoconus. A m J Ophthalmol 2003 May;135(5):620-27.

8. A car BT, Vural ET, A car S. Effects of contact lenses on the ocular surface in patients with keratoconus: Piggyback versus ClearK one hybrid lenses. Eye Contact Lens 2012 Jan;38(1): 43-48.

9. M CM ahon TT, Szczotka-Flynn L, B arr JT, et al. A new method for grading the severity of keratoconus: The keratoconus severity score (K SS). Cornea 2006 A ug;25(7):794-800.

10. Gordon M O, Steger-M ay K, Szczotka-Flynn L, et al. Baseline factors predictive of incident penetrating keratoplasty in keratoconus. A m J Ophthalmol 2006 Dec;142(6):923-30.

11. W ietharn BE, D riebe WT J r. Fitting contact lenses for visual rehabilitation after penetrating keratoplasty. Eye Contact L ens $2004 \mathrm{~J}$ an;30(1):31-33.

12. Ghosheh FR, Cremona F, A yres BD, et al. Indications for penetrating keratoplasty and associated procedures, 2001-2005. Eye Contact L ens 2008 J ul;34(4):211-14.

13. Geerards A , V reugdenhil W, K hazen A . Incidence of rigid gaspermeable contact lens wear after keratoplasty for keratoconus. Eye Contact L ens 2006 J ul;32(4):207-10.
14. Schirmbeck T, Paula JS, M artin LF, Crosio Filho H, Romao E. Efficacy and low cost in keratoconus treatment with rigid gaspermeable contact lens. A rq Bras Oftalmol 2005 M ar-A pr; 68(2):219-22.

15. L ema I, Duran JA, Ruiz C, Diez-Feijoo E, A cera A, M erayo J. Inflammatory response to contact lenses in patients with keratoconus compared with myopic subjects. Cornea 2008 A ug;27(7):758-63.

16. Gonzalez-M eijome J M , Jorge J, de A Imeida JB, Parafita M A. Soft contact lenses for keratoconus: Case report. Eye Contact Lens $2006 \mathrm{M}$ ay;32(3):143-47.

17. Schornack M M , Patel SV. Scleral lenses in the management of keratoconus. Eye Contact Lens 2010 Jan;36(1):39-44.

18. Pilskalns B, Fink BA, Hill RM . Oxygen demands with hybrid contact lenses. Optom V is Sci 2007 A pr;84(4):334-42.

\section{ABOUT THE AUTHOR}

\section{Gonzalo Carracedo}

Lecturer, Department of Optics II (Optometry and V ision), School of Optics, Complutense U niversity of Madrid, M adrid, Spain, e-mail: gonzalocarracedo@gmail.com 